\title{
(2) \\ Emerging Synergies Between Information Technology and Applied Ethnobotanical Research
}

\author{
Michael B. Thomas
}

\begin{abstract}
Ethnobotanical research has historically played a vital role in humankinds understanding of the relationship between people and the biological environment. Today, it remains a rapidly growing field of research, gaining professional, student and public interest both within the US and internationally. Ethnobiologists have however been very slow to adopt and apply tools of the informatics revolution and to integrate research data collaboratively. If ethnobotany is to continue to develop as a discipline, what is needed in the near future is not only a continued effort to promote collaborative ethnobotanical research but also to develop an initiative to bridge the digital gap between ethnobiologists and emerging bioinformatics tools. Through an improved understanding of the application of information technologies and the traditional ethnobotanical research model, tomorrow's scientists may better record and compare traditional botanical knowledge (TBK). This integration would greatly assist in stemming the tide of the unprecedented loss of global bio-cultural diversity in the twenty-first century.
\end{abstract}

\section{Introduction}

Of all the Earth's biological diversity, the plant kingdom is the most essential to human welfare and is extensively exploited for countless purposes including food, fuel, fiber, construction, tools, and medicine. Of these multiple uses, medicine is of high significance as plants have been by far the most important source of medicine for humankind throughout our evolutionary history. In broad terms, the study of the relationships between plants and people is known as ethnobotany. The American botanist John W. Harshberger coined the term "ethno-botany" in 1895 to describe studies of plants used by primitive and aboriginal people. His 1896 publication, "The Purposes of Ethnobotany", is generally accepted as a starting point for this field as an academic discipline (Harshberger 1896). Given the dual focus on plants and people, the ideal ethnobotanist receives training in a diverse array of interdisciplinary fields including anthropology, archeology, botany, chemistry, linguistics, psychology, ecology, pharmacology, law, and often requires the skills of both a diplomat and explorer (Balick and Cox 1996).

Today, ethnobotany remains an emerging discipline, one often difficult to characterize but generally concerned with the accelerating loss of both biological species and cultural diversity, specifically indigenous knowledge systems throughout the world. On one point however, there is agreement. Traditional knowledge systems are rapidly fading away. It is now widely recognized that as traditional cultures become increasingly Westernized, much of the richness of their traditions disappears (Balick and Cox 1996). Traditional Botanical Knowledge (TBK) that is often passed down orally to only select members of a community appears to be particularly susceptible to such cultural erosion. Urban drift, particularly of young generations, is a common contributing factor, as well as additional social problems such as warfare and famine, which often result in major displacement and disruptions of tra-

\section{Correspondence}

Michael B. Thomas, Centre for International Ethnomedicinal Education and Research, Gainesville, FL, U.S.A. mthomas@cieer.org

Ethnobotany Research \& Applications 1: 65-73 (2003) 
ditional societies. The problem is sometimes exacerbated by the neglect of local raw materials or manufactured items and the adoption of Western commercial products and health care modalities.

\section{Research Data and Collections}

Ethnobotanical collections and research data date back many centuries. But because data and collections are spread across many institutions and geographic regions they have been historically difficult to utilize and compare. Retrieving TBK research data has always been challenging because much of this information is published in disciplinary journals from diverse fields (e.g. botany, biology, anthropology, conservation biology) or in unpublished theses, dissertations, government documents and technical reports ("gray literature") that are not widely accessible. The diffuse distribution and variable quality of this data limit the ability of scientists to easily obtain access to either legacy data or current published ethnobotanical research.

A decade or two ago, a researcher interested in learning about the medical use of a particular plant species may have had to scan index cards, field notes and personal journals in order to find what had been collected, where and for what use. To learn what was contained in herbaria and museum collections a researcher had to physically visit them. This situation, however, is rapidly changing as a result of what has been described as the quiet revolution of information technology and bioinformatics (Bisby 2000). Today, an increasing amount of ethnobotanical research information is being stored electronically. In this paper, I will briefly summarize the current state of digital ethnobotanical documentation, review existing methods of electronic data storage and retrieval, present a recommendation for the development of a global information system using object-oriented design (OOD) technology and discuss the need for concerted action by the ethnobiological research community for its development.

\section{Bioinformatics Revolution in the Information Age}

Bioinformatics is a term so recently added to the scientific lexicon that it is featured in none of the latest dictionaries (Sugden and Pennisi 2000). "Bioinformatics" has gained popularity during the last few years to describe tools and techniques for storing, handling, and communicating the massive and ever increasing amounts of scientific (primarily biological) data. Made possible by dramatic improvements in computational power and computer accessibility, bioinformatics has become a major scientific discipline. Today, computers exceed human intelligence in a broad range of intelligent yet narrow domains such as playing chess, diagnosing certain medical conditions, buying and selling stocks, and guiding cruise missiles (Kurzweil 1999). In contrast, computers are still unable to describe photographs, objects on a museum table, write a summary of a movie, tie a pair of shoelaces, or perform other subtle tasks in which their human creators excel. One reason for this disparity in capabilities is that today's most advanced computer technology is still simpler than the human brain, currently about 1 million times simpler (Kurzweil 1999). But this disparity will not remain the case during the first half of the 21st century. Computer processing speed doubled every two years in the 1950s and 1960s, and is now doubling in speed every twelve months. This trend will continue, with computer technology achieving the memory capacity and computing speed of the human brain around 2020 (Kurzweil 1999).

Bioinformatics has principally emerged to provide a forum for the exchange of information in the fields of computational molecular biology and genome information. The emphasis has been on archiving, storage and retrieval of large amounts of research data and the development of databases capable of managing complex biological information generated by genome research. Applications in other fields are currently underway, and it has become clear that it is now essential that informatics technology be devoted to increasing our understanding of Earth's living diversity, and to develop new tools for archiving global diversity. The need for dynamically updated databases to support informed conservation decision-making is becoming increasingly recognized (Smith et al. 2000).

\section{Why the need for a collaborative authoritative global ethnobotanical database?}

Traditional approaches to gathering and disseminating ethnobotanical information are clearly inadequate to deal with the global increase of ethnobotanical research data. The print journal publication process is often very slow, and the relevant information (including large data sets, maps, and photographs) is often too large or complex for traditional print media. Electronic storage and retrieval offers an effective solution for the storage of data especially multimedia objects (digital images, sound, and video), comparative analysis, and the rapid distribution of a potentially large amount of data (Schalk and Oosterbroek 1996). Consequently, numerous electronic databases have recently been developed to disseminate information on plant uses. Most of these are available via the Internet through the World Wide Web (Table 1). However, because these databases were designed and developed independently, they are often oriented toward particular user groups (e.g. students, researchers, or the general public). As a consequence, the information they contain is very variable in its content and quality, often only having a regional or cultural focus.

Currently, the infrastructure for collaborative interdisciplinary scientific research through the Internet is growing, one rich with power and promise (Lucky, 2000). However, many web sites delivering ethnobotanical information contain anecdotal data and questionable research meth- 
Table 1.0 Current Digital Ethnobotanical Databases.

\begin{tabular}{|c|c|}
\hline A Modern Herbal & www.botanical.com/botanical/mgmh/mgmh.html \\
\hline A Guide to Medicinal and Aromatic Plants & www.hort.purdue.edu/newcrop/med-aro/default.html \\
\hline AGIS, EthnobotDB & permanently decommissioned on April 15, 2002. \\
\hline AGIS, Medicinal Plants of Native America & permanently decommissioned on April 15, 2002. \\
\hline AGIS, Native American Food Plants & permanently decommissioned on April 15, 2002. \\
\hline APINMAP - An Asian Medicinal Plants Database & CD only (out of print) \\
\hline Botanical Dermatological Database & www.bodd.cf.ac.uk \\
\hline Brunei Dusun Database & lucy.ukc.ac.uk/brunei.html (Filemaker Pro download) \\
\hline Ethnobotany of the Peruvian Amazon & www.biopark.org/Plants-Amazon.html \\
\hline Ethnoecology Database of the Greater SW & www.anthro.fortlewis.edu/ethnobotany/database.htm \\
\hline Florin Medicinal Plants - Russia & www.florin.ru/eng/index_eng.html \\
\hline Herbage, 4th Edition & www.holisticopia.com/herbs/ \\
\hline Indonesian Folk Medicine & www.public.iastate.edu/ CYBERSTACKS/hyb_r_2.htm \\
\hline Mayan Ethnomedical Encyclopedia & www.ecosur.mx \\
\hline MEDFLOR (MEDicinal FLORa) & Not available online - Limited access \\
\hline Medicinal Plants of Ibiza, Spain & CD ROM only \\
\hline Medicinal Plants of Madagascar & www.lunerouge.org/ilerouge/produits/prod01_e.htm \\
\hline Medicinal Plants of the Quijos - Quichua Shamen & www.public.iastate.edu/ cbutter/ethnofra.htm \\
\hline Medicinal Uses of Native Plants of South Florida & www.fig.cox.miami.edu/7Escofield/sofl_plants/med_index.html \\
\hline MedPlant Pharmdatabase & www.medplant.mahidol.ac.th/index.asp \\
\hline NAPRALERT & www.info.cas.org/ONLINE/DBSS/napralertss.html \\
\hline Native American Ethnobotany Database & herb.umd.umich.edu \\
\hline Natural Medicines Comprehensive Database & www.naturaldatabase.com \\
\hline Natural Products Database & www.naturalproducts.org \\
\hline Online Directory for Medicinal Plant Conservation & www.genres.de/Mpc-dir/ \\
\hline Phytochemical and Ethnobotanical Databases & www.ars-grin.gov/duke/ethnobot.html \\
\hline Plantas Medicinais (CD ROM only) & www.agromidia.com.br/plantas_completo.htm \\
\hline Plants For A Future & www.comp.leeds.ac.uk/pfaf/index.html \\
\hline Plants of the Machiguenga, PRELUDE & www.preludedb.be.tf/ \\
\hline PROSEA & www.prosea.nl \\
\hline PROTA & www.prota.org \\
\hline SEPASAL & www.rbgkew.org.uk/ceb/sepasal/ \\
\hline State Administra. of Traditional Chinese Medicine & www.cintcm.ac.cn/jsxt-e.html \\
\hline TCM Herb Database & www.rmhiherbal.org/ai/pharintro.html\#herbdb \\
\hline Tibetan Medicinal Plants & $\begin{array}{l}\text { www.home.t-online.de/home/520097278994-0001/yuthog/frameset/ } \\
\text { plants.html }\end{array}$ \\
\hline The American Herbal Pharmacopoeia & www.herbal-ahp.org/mono.html \\
\hline The Gatherer \& Plant Use Search Engine & www.kippewa-gardens.com/html/main/gatherer.html \\
\hline Traditional Samoa Medicinal Plants & www.dittmar.dusnet.de/english/ \\
\hline TradiMed & www.tradimed.com/index.asp \\
\hline TRAMIL & www.funredes.org/tramil/english/ \\
\hline
\end{tabular}


odology and often do not include the detailed references necessary for sound scientific research. Even though scientists are, as a group, comfortable with computers and information networks, they generally have been slow to provide online access to their research. Nonetheless, with Internet traffic doubling every 6 months, and wireless capacity doubling every 9 months the value of using current information technology for both storing and retrieving ethnobotanical research data for a variety of purposes has become increasingly apparent.

\section{Current Internet Databases and Digital Resources}

Ethnobotanical information is increasingly being recorded in databases and delivered via the Internet. Table 1 lists some of the databases that are available. This list is not exhaustive because I am undoubtedly not aware of all electronic databases that are currently available. The term "database" has been used broadly to describe many kinds of collections. For example, current online databases vary from facsimile replications of Gerard's Herbal (1633) to Herculean efforts such as HerbWeb.com (Johnson 2000) developed by compiling published medicinal plant research data using conventional relational database technology. Existing plant databases delivered via the Internet can be roughly categorized into (1) facsimile reproductions of printed materials, including Adobe PDF files (2) listings of medicinal plants and some of their properties as HTML-based web page "databases", similar to Russo's Plants of the Machiguenga website (Russo 2000) (3) downloadable spreadsheets (4) downloadable relational database tables often using Microsoft Access or FileMaker Pro files, such as Ellen's downloadable Brunei Dusun database (Ellen 2000) (5) online relational databases that can be searched by a Web interface, such as the Native American Ethnobotany database by Moerman (Beckstrom-Sternberg et al. 1995, Moerman 1999) and the Worldwide Ethnobotany database developed by Duke and Beckstrom-Sternberg (Beckstrom-Sternberg et al. 1994) and (6) object-oriented databases (OODB) such as the one proposed in this paper.

Additionally, the notion of scientific web portals and digital libraries have emerged and are becoming popular. These are single web sites that serve as entrances to integrate material in a particular field similar to popular portals such as Google or Yahoo!. A good example of this concept is EcoPort (EcoPort 2000), which aims to be a one-stop encyclopedia of information on every known plant and animal. EcoPort was developed to bring together information that is currently scattered across the internet on individual researcher's or university's Web sites and stores all its data in one database that is accessed through one site. Similarly, NAPRALERT (Gyllenhaal et al. 1993) is a fee-based system which compiles information on natural products (chemistry, medicinal plant folklore, and biologi- cal activities) from many published sources. Both EcoPort and NAPRALERT use centralized relational database technology.

In contrast to these centralized approaches, meta-database search engines such as The Gatherer (Kippewa Gardens 2000) or the Species Analyst (Species Analyst 2000) search and gather data from numerous online botanical databases. These use various techniques, including fulltext search, Z39.50 common database interface standards (Z39.50 2000), and XML (XML 2000) for data retrieval and exchange. They work either with unstructured text data or minimal models of the record structure in the underlying databases that they search.

Among the first and largest international collections of plant and animal species information, Species 2000 (Bisby et al. 1993, Bisby 2000, Species 2000) focuses on the problem of developing taxa for the world's collections of biodiversity information. Species 2000 also acts as a meta-database by pointing to other, locally maintained databases built within the Species 2000 framework. Scientific collaborators worldwide integrate their information into the Species 2000 data model. The key to this collaboration consists of dividing global taxonomic classifications vertically so that individual projects don't conflict as much as they would across horizontal, and flora studies. The plan for building a unified ethnobotanical database partially overlaps with other larger database projects such as the Integrated Taxonomic Information System (ITIS), Species 2000 or the Tree of Life (The Tree of Life Web Project 2001) and some sharing can occur, especially in the area of plant taxonomy.

Although the current ethnobotanical databases are of great value in promoting the awareness of the need to record and conduct ethnobotanical research, they are insufficient, especially for collaborative and comparative analysis. I suggest that a coordinated global approach is necessary to currently manage the increasing amount of bio-cultural knowledge being documented by ethnobiologists worldwide. The growing number of independent ethnobotanical databases is itself a compelling argument for a unified coordinated data management system. A coordinated unified database linked to independently managed databases already available on the Internet, with the individual databases remaining distributed is envisioned. The system's primary goals would be to function as both a comprehensive digital library with a search engine for retrieving information on the present, past and future uses of plants, providing a "one-stop educational resource" for users. As new technologies for data retrieval are developed, the system could evolve into a meta-database - that is, a system that allows researchers to analyze comparative data pooled from multiple databases. However, before any initiative begins it is vital that a standard lexicon of terms and a unified methodology of recording TBK be designed, developed and embraced by the potential us- 
ers. It is my contention that such an initiative could begin with the initial development of a standardized method of archiving TBK concerning the use of plant medicines with the long-term goal being to build a common digital library of use categories or classes of ethnobotanical information.

\section{Developing a Standard Unified Methodology for Ethnomedicinal Research}

Ethnobotanical studies involving the identification of plant species used as medicines by many individual cultures has long been an active area of research (Berlin and Berlin 1996, Johns 1990, Moerman et al. 1999). In the past century many scholarly books, papers and numerous journal articles have been published to support the inescapable fact that plants used by humans, many being used primarily by indigenous peoples, have been identified, researched and eventually developed as the source of some of the world's most important pharmaceuticals. However, few studies have sought to create an iterative compilation of recorded plant uses or a unified approach to relate the use of medical plant species to phylogeny in order to gain a greater understanding of the kinds or characteristics of plants which are more or less important (Moerman 1991, Moerman 1996, Phillips and Gentry 1993). The lack of a unified approach has also led to a paucity of comparative ethnobotanical studies, studies that not only examine different uses of the same types of plants in different cultures, but also compare the manner in which these plants figure in different worldviews (Balick and Cox 1996). Few papers published in the leading ethnobotanical journals, Economic Botany, the Journal of Ethnopharmacology, Journal of Ethnobiology, and the Journal of Pharmaceutical Biology, comprehensively grapple with comparative analysis of plant uses across cultures. Almost nothing is known about possible patterns of medicinal plant selection by human beings across cultures, regions and hemispheres (Moerman et al. 1999).

Traditionally, ethnobotanical researchers have worked independently, and in many cases largely unaware, of each other's research. They have tended to be isolated by their differences in education, methodology, and knowledge of the applications of computer technology. Recently, these artificial barriers to communication have been breached, and increasingly ethnobotanists are beginning collaborative research to explain the patterns and process of plant use across a broad range of spatial and temporal scales. Moerman et al. (1999) have suggested that the similarity of medicinal plant use implies that human knowledge is deeply rooted. While it seems unlikely that there is a continuous web of knowledge of individual species use back to the middle Paleolithic, Moerman et al. (1999) suggest that non-Western peoples speaking unrelated languages have somehow generated similarly useful knowledge over broad geographic regions, or possibly carried the knowledge of medicinal plants with them throughout Asia and to America during migrations since the Upper Paleolithic, or both. Recognizing such patterns could increase the efficiency of contemporary search for novel phytochemicals at the same time as they demonstrating the existence of a global pattern of human knowledge (Moerman et al. 1999).

The standardization of terms and a unified system to describe and record medicinal plant uses would be of enormous benefit to researchers, especially where exchanges of data sets are involved (Cook 1995). One such standard is the Economic Botany Standard developed by the International Working Group on Taxonomic Databases for Plant Sciences (TDWG) (Cook 1999), which has been adopted as a standard by the International Union of $\mathrm{Bi}$ ological Sciences (IUBS) Taxonomic Databases Working Group. This standard provides a mechanism, whereby, uses of plants (in their cultural context) can be described, using standardized descriptors and terms, and then attached to taxonomic data sets. However, attempts to utilize the standardized schema have not been widely implemented nor fully realized for several reasons. First, the Economic Botany Data Collection Standard (EBDCS) is not very flexible because it does not allow users to extend the range of terms beyond what is defined, and it also contains inconsistencies (Cook 1999). Second, implementing the standard into a relational format is awkward (Cook 1999). Finally, it was designed not as a single unifying database but rather as a tool for potential users to in development of independent economic botany databases. The Economic Botany Subgroup of the Taxonomic Development Working Group, has recently evaluated the extent to which the EBDCS meets the needs of users and what revisions and additional data standards would be useful (TDWG 2003). More discussion on its development is planned in the future.

In contrast to the Economic Botany Data Collection Standard, a new proposal for a database model would be based on Object Oriented Design (OOD) technology (Barry 1996), which is capable of storing concepts and knowledge as well as raw data. This new approach uses data structures called objects to represent concepts. In contrast to traditional, established databases based on the relational model in which data are viewed as records, object databases provide a more natural description of the entities within a domain by examining objects, their properties, and interrelationships. Data modeling simply involves studying a concept and creating a symbolic representation of that concept. The symbolic representation captures the meaning of the concept and is a data structure that can be stored and manipulated in a computer. Traditionally databases are used to store raw field observations and other facts prior to analysis, and current Web site "databases" simply arbitrarily store knowledge in unstructured HTML. In addition, Object-oriented data models are more dynam- 
ic and the glossary or terms or lexicon can be extended to include specific terms identified or suggested by individual researchers. Consequently, researchers have the ability to actively contribute their expertise and experience to improve the design of the data model.

Standards such as the Economic Botany Standard can however, serve to bootstrap a glossary of terms within a particular domain. The Economic Botany Standard can readily be incorporated into the linguistic portion of the proposed data model as a starting point for covering economic botany terminology. The taxonomic structure of the object-oriented data model easily supports the existing structure of this standard without having to worry about mapping into relational tables.

\section{The Application of Object Oriented Databases in Ethnobotanical Research}

Object Oriented Databases (OODB) provide persistent storage for objects. They provide all the features of conventional relational database management systems. In addition, they provide an intuitive method to visually represent knowledge systems through data modeling. Data modeling is a tool which can assist researchers in representing the world as being a collection of things. These things are objects. Thus people, plants, diseases, populations, pictures, concepts, documents, equations, maps, and decision rules are all objects. Everything can be represented as objects. Objects have both state (properties) and behavior. For example, a plant has properties such as a color, scientific name, reproductive strategy, and use. The plant can grow, reproduce, disperse, and exhibit other behavior. Objects having similar properties and behavior are grouped into a common classes (e.g. Green Plants, Medicinal Plants and Food Plants are all Eukaryotes) (Figure 1).

Figure 1. Relationship between Superclass, Class and Objects

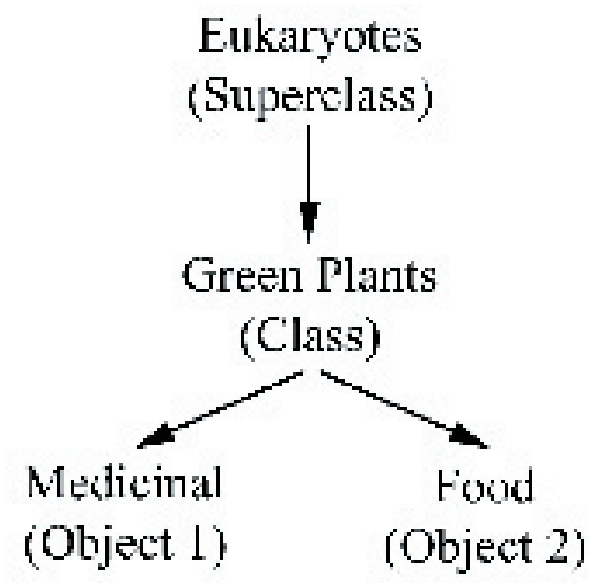

Objects can have an extremely complex structure (so they can model complex things in the world such as medical knowledge systems). Figure 2 provides an example of how objects can be stored using an OODB. The figure shows an example of the relationship between an observation or instance of the herbarium voucher MT 987 of an identified medicinal plant. The plant instance is composed of other objects (Ecology, Economic Botany, Plant Systematics and Pharmacology). The figure also shows the relationship of how these objects are interconnected. The representation of nodes and links can also be visually incorporated by the development of a graphical user interface which could dynamically illustrate these relationships.

In contrast a relational database (Oracle, SQL Server, MySQL, Microsoft Access), data is represented using tables with rows describing individual data items, and columns defining data attributes. These databases use SQL (Structured Query Language) as the universal language for querying relational databases. However, not all ethnobotanical data can be stored conveniently in tables. ODBMS provide a new model for organizing data as complex objects. ODBMS can stores complex data types (text, multimedia, equations, applications) and can integrate large, diverse, heterogeneous collections of information. Finally, ODBMS provides formalism for building large knowledge bases or digital libraries (Beck and Xin 1998). Most pure ODBMS packages provide a data interface to relational databases, and therefore can transparently incorporate the data stored in existing relational database into the object database.

The proposed Object Oriented database model (OODB) and associated data contribution mechanism would provide researchers with the ability to utilize shared research data and to initiate more collaborative and comparative analysis. Having the ability to recognize patterns of plant use, supported by the OODB model, may increase the efficiency of contemporary drug discovery programs for useful natural products and at the same time provide increasing support for documenting global patterns of both independent and shared human knowledge (Moerman et al. 1999).

The proposed approach would use a common extensible data model and provide tools to researchers to develop a global database. In this way it is similar to EcoPort (EcoPort 2000) but with a more complex data model. The approach is logically centralized because a common data model is used. But it can be physically distributed, because it is possible to have many subsets of the database existing in different places. In contrast, many systems (Species 2000, Species Analyst 2000, Kippewa Gardens 2000) assume that researchers will develop their own local databases, and provide a standard interface to higherlevel meta-databases so that their data can be searched globally. There are pros and cons to both approaches. A 
Figure 2. Example of an Object-oriented Database illustrating relationships between an observation or instance of the herbarium voucher MT 987 and other objects.

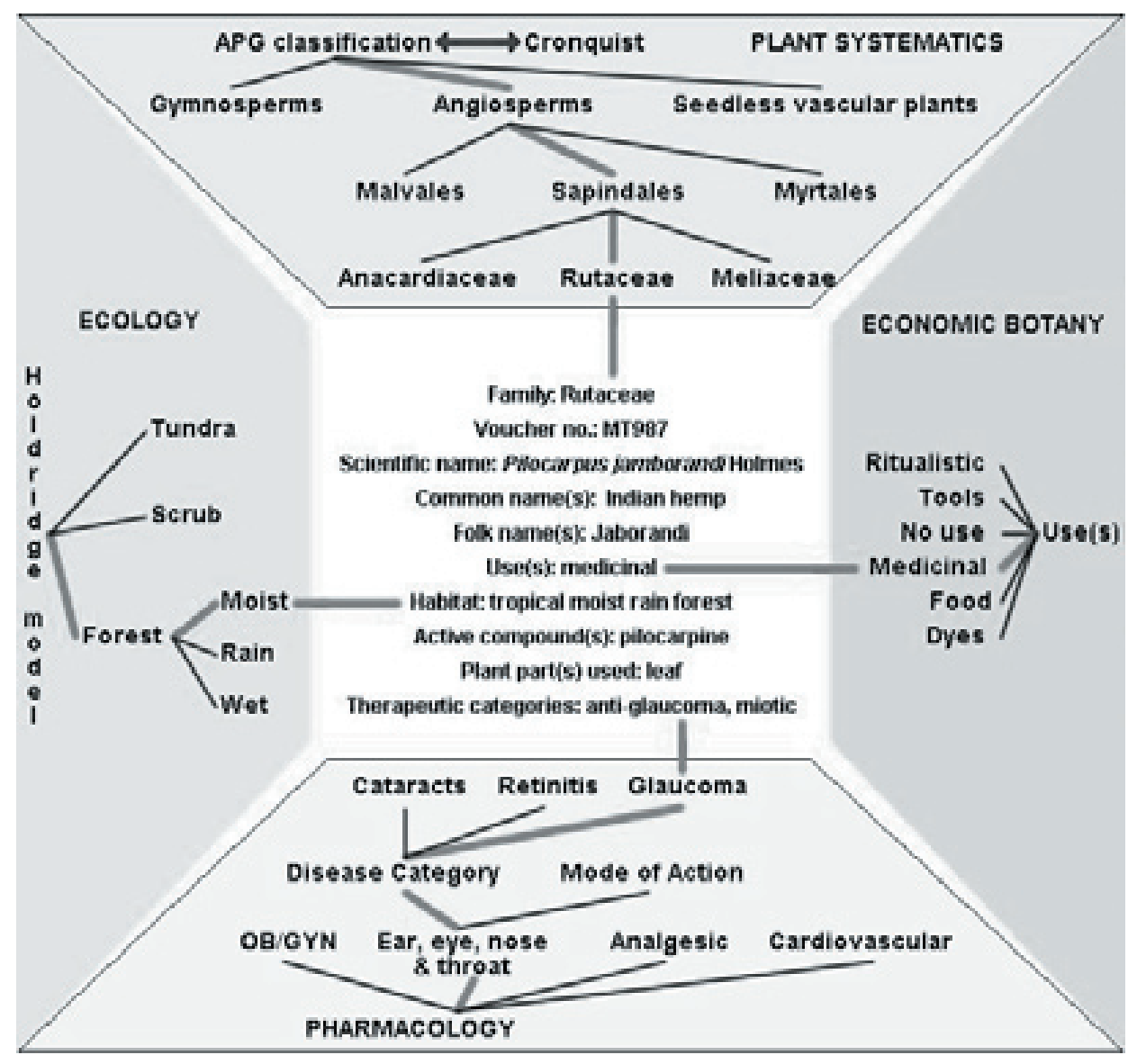

compromise might be to use the OODB model to describe the contents of existing databases (such as legacy data) and thus it can serve both as a central repository and a meta-database, with a richer modeling language. Nonetheless, what is needed now is a data model which can serve as starting point for building an OODB and the first step is to develop a robust data model.

\section{Conclusion}

Equipped with new scientific tools from molecular biology, analytical chemistry, mechanical engineering, and medical anthropology, modern ethnobotanists are asking a dazzling array of novel questions while shedding new light on older questions. Yet, the lack of a unified approach and standard data model for recording research data has led to a paucity of comparative ethnobotanical studies studies that not only examine different uses of the same type of plants in different cultures, but also compare the ways plants figure in different world views (Balick and Cox 1996). Few papers published by leading scientific journals address comparative analysis of possible patterns of medicinal plant selection and use by humans across cultures, regions or hemispheres. Indeed the absence of readily accessible comparative sources of ethnobotanical data has been recognized as a serious hindrance (Thomas at al. 2001).

While the amalgamation of many different educational backgrounds and disciplines has enriched the field of ethnobotany, the lack of a clear consensus on such basic issues as disciplinary goals, data management and archiving methodologies has hampered the development of a unified and collaborative approach (Balick and Cox 1996, Thomas et al. 2001). The OODB model not only satisfies the need for a tool for archiving TBK but may also provide the foundation for a centralized globally current data resource. A dynamic resource would encourage a unified approach by facilitating a greater opportunity for comparative analysis of research data through direct contributions by members of the ethnobiological research community. The description of a new data model for ethnobotanical data based on the OODB model has been suggested and shown how it can be applied to several areas (medical terminology, systematics, ecology, and pharmacology) resulting in an integrated, cross-disciplinary 
database (Thomas 2001, Thomas et al. 2001). Tools to assist researchers in browsing and editing the database can also been developed. The next steps would include expanding the data model to cover additional disciplines (such as economic botany), and accessing information from existing databases and resources either by importing data or creating metadata descriptions of these resources. It is suggested that an international TBK standards committee would ultimately need to be formed within the ethnobiology community, as has been done successfully for other global databases such as the Tree of Life or IT IS web projects, with the framework for a global ethnobotanical information system being based on the OODB model.

\section{References Cited}

Angiosperm Phylogeny Group. 1998. An Ordinal Classification for the Families of Flowering Plants. Annals of the Missouri Botanical Garden 85: 531-553.

Balick, M.J. and P. A. Cox. 1996. Plants, People, and Culture: The Science of Ethnobotany. Scientific American, New York. 256 pp.

Barry, D.K. 1996. Object Database Handbook: How to Select, Implement, and Use.Object-Oriented Databases. John Wiley \& Sons, New York.

Beck, H.W. and J.N. Xin. 1998. Using Java, CORBA, and ODBMS to develop agricultural databases. in Proceedings of the 7th International Conference on Computers in Agriculture. Orlando, Florida.

Beckstrom-Sternberg, S.M., J.A. Duke, and K.K. Wain. 1994. The Ethnobotany Database. (Data version July 1994). www.ars-genome.cornell.edu/cgi-bin/WebAce/

Beckstrom-Sternberg, S.M., D.E. Moerman and J.A. Duke. 1995. The Medicinal Plants of Native America Database. (Data version June 1995). www.ars-genome.cornell.edu/cgi-in/WebAce/

Berlin, L. and B. Berlin. 1996. Medical Ethnobiology of the Highland of Maya, Chiapas, Mexico: The Gastrointestinal Diseases. Princeton University Press, Princeton.

$591 \mathrm{pp}$

Bisby, F. A. 2000. The Quiet Revolution: Biodiversity Informatics and the Internet. Science 289:2309-2312.

Bisby, F. A., G. F. Russel, and R. J. Pankhurst. 1993. Editors of Designs for a Global Plant Species Information System. Oxford Science Publications, Oxford.

Cook, F.E.M. 1995. Economic Botany Data Collection Standard. Prepared for the International Working Group on Taxonomic Databases for Plant Sciences (TDWG). Kew: Royal Botanic Gardens, Kew.

Cook, F.E.M. 1999. Economic Botany Data Collection Standard. www.rbgkew.org.uk/tdwguses/index.htm

EcoPort. 2000. www.ecoport.org

Ellen, R. 2000. Brunei Project - Databases. lucy.ukc. ac.uk/brunei.html

Gerard, J. 1633. The Herball, or, Generall Historie of Plantes. London: A. Islip, J. Norton, and R. Whitakers.

Gyllenhaal, C. Quinn, M.L., Soejarto, D.D. and N.R. Farnsworth. 1993. NAPRALERT: problems and achievements in the field of natural products. In Designs for a Global Plant Species Information System. Edited by F.A. Bisby, G.F. Russell, and R.J. Pankhurst. Oxford University Press, Oxford.

Harshberger, J.W. 1896. The purposes of ethno-botany. Botanical Gazette 21:146-154.

Johns, T. 1990. With Bitter Herbs They Shall Eat It: Chemical Ecology and the Origins of Human Diet and Medicine. University of Arizona Press, Tucson. 356 pp.

Johnson, T. 2000. Herbweb. Global Botanical Exchange. www.herbweb.com

Kippewa Gardens. 2000. The Gatherer. Plant Use Multiple Database Search Engine. www.kippewa-gardens. com/cgi-bin/Gatherer.pl

Kurzweil, R. 1999. The Age of Spiritual Machines: When Computers Exceed Human Intelligence. Viking Press, New York.

Lucky, R. 2000. The Quickening of Science Communication. Science. 289: 259-264.

Moerman, D. 1991. The Medicinal Flora of Native North America: An Analysis. Journal of Ethnopharmacology $31: 1-42$

Moerman, D. 1996. An Analysis of the Food Plants and Drug Plants of the Native North America. Journal of Ethnopharmacology 52(1):1-22.

Moerman, D., R.W. Pemberton, D. Kiefer, and B. Berlin.1999. A Comparative Analysis of Five Medicinal Floras. Journal of Ethnobiology 19(1): 49-67.

Phillips, O. and A. Gentry. 1993. The Useful Plants of Tambopata, Peru: I. Statistical hypothesis tests with a new quantitative technique. Economic Botany 47:15-32. 
Russo, E.B. 2000. In His Footsteps: The Machiguenga. Wise Traditions. Food, Farming and the Healing Arts 1:4345.

Schalk P.H., and P. Oosterbroek. 1996. Interactive knowledge systems: Meeting the demand for disseminating upto-date biological information. Biodiversity Letters 3:119123.

Smith, T., L. Biotani, C. Bibby, D. Brackett, F. Corsi, G.A.B. da Fonesca, C. Gascon, M.G. Dixon, C. Hilton-Taylor, G. Mace, R.A. Mittermier, J. Rabinovich, B.J. Richardson, A. Rylands, B. Stein, S. Stuart, J. Thomsen, and C. Wilson. 2000. Databases Tailored for Biodiversity Conservation. Science 289:2073.

Species 2000. www.sp2000.org

Species Analyst. 2000. www.habanero.nhm.ukans.edu

Sugden, A. and E. Pennisi. 2000. Diversity Digitized. Science 289:2305.

TDWG. 2003. Economic Botany Subgroup Report, October 1999. Taxonomic Databases Working Group. www. tdwg.org/botany.html

Tree of Life Web Project. 2001. tolweb.org/tree/

Thomas, M.B. 2001. An Analysis of the Pataxó Pharmacopoeia of Bahia, Brazil, Using an Object Oriented Database Model. Dissertation. University of Florida, Gainesville.

Thomas, M.B., N. Lin. and H.W. Beck. 2001. A Database Model for Integrating and Facilitating Collaborative Ethnomedicinal Research. Pharmaceutical Biology. 39:Supplement:41-52.

XML. 2000. www.w3.org/XML

Z39.50. 2000. www.loc.gov/z3950/agency 